\title{
Multi-resource scheduling of an FMC in discrete parts manufacturing
}

\author{
Geatse Meester and Henk Zijm \\ University of Twente, The Netherlands
}

In this paper we develop a scheduling procedure for a Flexible Manufacturing Cell (FMC), consisting of a number of basically identical machining centres and operating as part of a larger component manufacturing shop (basically a hybrid job shop). Each job on the FMC is subject to release and due dates; the objective of the scheduling procedure is to minimize the maximum lateness, subject to both machine and cutting tool capacity constraints.

An important aspect of the system is the presence of a tool robot which can interchange tools between a central tool store and the local tool magazines dynamically, i.e. during machine operations. However, since many cutting tools are unique (i.e. no duplicates are present in the system) the scheduling problem is multi-resource in nature: both machines and tools are scarce resources which have to be used simultaneously. A heuristic to solve this problem is proposed and test results are presented for both, theoretical tests and a test with an FMC in a company producing pneumatic and electrical actuator valves. 